\title{
Bernoulli actions are weakly contained in any free action
}

\author{
Miklós Abért and Benjamin Weiss
}

September 28, 2011

\begin{abstract}
Let $\Gamma$ be a countable group and let $f$ be a free probability measure preserving action of $\Gamma$. We show that all Bernoulli actions of $\Gamma$ are weakly contained in $f$.

It follows that for a finitely generated group $\Gamma$, the cost is maximal on Bernoulli actions for $\Gamma$ and that all free factors of i.i.d. of $\Gamma$ have the same cost.

We also show that if $f$ is ergodic, but not strongly ergodic, then $f$ is weakly equivalent to $f \times I$ where $I$ denotes the trivial action of $\Gamma$ on the unit interval. This leads to a relative version of the Glasner-Weiss dichotomy.
\end{abstract}

\section{Introduction}

Let $\Gamma$ be a countable group. By a probability measure preserving (p.m.p.) action $f$ of $\Gamma$ we mean a representation of $\gamma \in \Gamma$ by $f_{\gamma}$ in the group of measure preserving transformations of $(X, \mathcal{B}, \mu)$. Here $(X, \mathcal{B}, \mu)$ is a standard Borel probability space. The action $f$ is free, if for $\mu$-almost every $x \in X$ and $\gamma \neq \gamma^{\prime} \in \Gamma$ we have $f_{\gamma}(x) \neq f_{\gamma^{\prime}}(x)$.

Let $f$ and $g$ be p.m.p. actions of $\Gamma$ on $(X, \mathcal{B}, \mu)$ and $(Y, \mathcal{C}, \nu)$, respectively. Following the definition of Kechris [7, we say that $f$ weakly contains $g(f \succeq g)$ if for all Borel subsets $Y_{1}, \ldots, Y_{n} \in \mathcal{C}$, finite sets $S \subseteq \Gamma$ and $\varepsilon>0$ there exist Borel subsets $X_{1}, \ldots, X_{n} \in \mathcal{B}$ such that

$$
\left|\mu\left(f_{\gamma} X_{i} \cap X_{j}\right)-\nu\left(g_{\gamma} Y_{i} \cap Y_{j}\right)\right|<\varepsilon \quad(1 \leq i, j \leq n, \gamma \in S) .
$$

In particular, the way $g$ acts on finite partitions of the underlying space can be simulated by $f$ with arbitrarily small error. A special case of weak containment is when $g$ is a factor of $f$, that is, there exists a surjective measure preserving $\Gamma$-equivariant map from $(X, \mathcal{B}, \mu)$ to $(Y, \mathcal{C}, \nu)$. We call $f$ and $g$ weakly equivalent if $f \succeq g$ and $g \succeq f$.

Let $\kappa$ be a probability space. The Bernoulli action $\kappa^{\Gamma}$ is defined as the set of maps from $\Gamma$ to $\kappa$, endowed with the product measure and the shift action by $\Gamma$. These actions are also called i.i.d. processes on $\Gamma$. 
The main result of this paper is the following:

Theorem 1 Let $\Gamma$ be a countable infinite group and let $f$ be a free p.m.p. action of $\Gamma$. Then $f$ weakly contains every Bernoulli action of $\Gamma$. In particular, all free factors of i.i.d.-s of $\Gamma$ are weakly equivalent.

Note that one can obtain the original Rokhlin lemma for $\mathbb{Z}$ as a quick corollary of Theorem 1. In this language, the lemma says that every free action of $\mathbb{Z}$ weakly contains the cyclic action of $\mathbb{Z}$ on $n$ points for all $n>0$. Since weak containment is transitive, by Theorem [1, it is enough to prove this for $\{0,1\}^{\mathbb{Z}}$ with $p(0)=p(1)=1 / 2$. For a natural number $k, 0 \leq l \leq n-1$ and $\omega \in\{0,1\}^{\mathbb{Z}}$ let

$$
\|\omega\|_{k, l}=\sum_{i=0}^{k-1} \omega(i n+l)
$$

and let

$$
A_{k}=\left\{\omega \in\{0,1\}^{\mathbb{Z}} \mid\|\omega\|_{k, 0}>\|\omega\|_{k, l}+n \text { for all } 1 \leq l \leq n-1\right\} .
$$

Let $T$ denote the shift operator on $\{0,1\}^{\mathbb{Z}}$. Then the sets $A_{k}, T A_{k}, \ldots, T^{n-1} A_{k}$ are disjoint, while the measure of $A_{k}$ converges to $1 / n$ as $k$ tends to infinity, since for a fixed $k$, the $\|\omega\|_{k, l}$ are independent binomial variables.

As a corollary of Theorem 1, one gets a new result on cost. The cost is a numeric invariant of a p.m.p. action of a countable group. Its study was initiated by G. Levitt $[8$ and has been investigated in depth by D. Gaboriau [3. One of the major open problems here is the Fixed Price problem, that is, whether every free action of a countable group has the same cost. A. Kechris showed that for finitely generated groups and free actions, the cost is monotonic with respect to weak containment [7, Corollary 10.14], so Theorem 1 leads to the following.

Corollary 2 Let $\Gamma$ be a finitely generated group. Then, among free p.m.p. actions of $\Gamma$, the cost is maximal on free factors of i.i.d.-s. In particular, all free factors of i.i.d.-s have the same cost.

Since the cost of any free action of an infinite group is at least 1 , we get that an infinite group $\Gamma$ has fixed price 1 if and only if one of its Bernoulli actions have cost 1 . We will generalize the monotonicity result of A. Kechris to actions that are not necessarily free, but for that we need to use an extension of the notion of cost called the groupoid cost. For details see Section 3

In the language of weak containment, one can express the well-known notion of strong ergodicity as follows. An action of $\Gamma$ is strongly ergodic, if it is ergodic and it does not weakly contain the identity action of $\Gamma$ on two points with equal mass.

In the presence of ergodicity, the absence of strong ergodicity forces various structural restrictions on the action. An example of this is the theorem of V. Jones and K. Schmidt [6] that says that the equivalence relation defined by such 
an action factors onto a nontrivial hyperfinite equivalence relation. We will give another kind of restriction in the language of weak containment.

For a measure preserving action $f$ on $(X, \mathcal{B}, \mu)$ let $f \times I$ denote the diagonal action of $\Gamma$ on $(X, \mathcal{B}, \mu) \times[0,1]$ where $\Gamma$ acts on the second coordinate trivially.

Theorem 3 Let $f$ be an ergodic p.m.p. action of a countable group. Then the following are equivalent:

1) $f$ is not strongly ergodic;

2) $f$ is weakly equivalent to $f \times I$.

This leads to the following convexity result, that can be viewed as a 'relative Glasner-Weiss theorem'.

For a compact topological space $K$ let $K^{\Gamma}$ denote the shift of $\Gamma$ with base set $K$. This is a continuous action on a compact space. Let $M(\Gamma, K)$ denote the set of $\Gamma$-invariant Borel measures on $K^{\Gamma}$, endowed with the weak* topology and let $E(\Gamma, K) \subseteq M(\Gamma, K)$ denote the set of ergodic measures. It is a standard fact that $M(\Gamma, K)$ is a simplex where the set of extreme points equals $E(\Gamma, K)$.

For an ergodic p.m.p. action $f$ on $(X, \mathcal{B}, \mu)$ any Borel map $\phi: X \rightarrow K$ defines a $\Gamma$-equivariant map $\Phi: X \rightarrow K^{\Gamma}$ by setting, for $x \in X$ and $\gamma \in \Gamma$

$$
\Phi(x)(\gamma)=\phi\left(f_{\gamma}(x)\right) .
$$

The measure $\Phi \circ \mu$ is an invariant measure on $K^{\Gamma}$.

Let

$$
E(f, K)=\{\Phi \circ \mu \mid \phi \text { a Borel map from } X \text { to } K\} .
$$

One can also describe $E(f, K)$ as the set of invariant measures on $K^{\Gamma}$ that are factors of $f$. We have the following dichotomy, that can be viewed as a 'relative Glasner-Weiss theorem'.

Theorem 4 Let $f$ be an ergodic p.m.p. action of the countable group $\Gamma$. If $f$ is strongly ergodic, then $\overline{E(f, K)} \subseteq E(\Gamma, K)$. If $f$ is not strongly ergodic, then $\overline{E(f, K)}$ is convex.

The Glasner-Weiss theorem [5] says that when $\Gamma$ has property $(\mathrm{T})$, then $E(\Gamma, K)$ is closed, and when it does not have property $(\mathrm{T})$, then $\overline{E(\Gamma, K)}=$ $M(\Gamma, K)$. We can deduce this result from Theorem 4 as follows. By the GlasnerThouvenot-Weiss theorem 4, for every countable group $\Gamma$ there exists an ergodic p.m.p. action $f$ of $\Gamma$ that weakly contains all ergodic actions of $\Gamma$. This means that $E(\Gamma, K) \subseteq \overline{E(f, K)}$.

If $\Gamma$ has property $(\mathrm{T})$, then by K. Schmidt [10, every ergodic action of $\Gamma$ is strongly ergodic, in particular, $f$ is strongly ergodic. So by Theorem 4 $\overline{E(f, K)}=E(\Gamma, K)$ and hence $E(\Gamma, K)$ is closed.

If $\Gamma$ does not have property ( $\mathrm{T}$ ), then by the Connes-Weiss theorem, $\Gamma$ has an ergodic action that is not strongly ergodic. But that action is weakly contained in $f$, so $f$ is also not strongly ergodic. So by Theorem $4, \overline{E(f, K)}$ is convex and since it contains all of $E(\Gamma, K)$, it must equal $M(\Gamma, K)$, the convex hull of $E(\Gamma, K)$. 
The paper is organized as follows. In Section [2 we prove Theorem [1, In Section 3 we define the groupoid cost and show that for finitely generated groups, it is monotonic with respect to weak containment. Finally, in Section 4 we prove Theorem 3 and Theorem 4 .

Acknowledgement. The authors thank the Glasner family (Eli and Yair) for establishing a link between them, that led to this paper.

\section{Weak containment of i.i.d. actions}

In this section we prove Theorem 1. Throughout the paper, when it is convenient, we will assume that the p.m.p. action is continuous on a compact metric space. This can be easily seen by taking a Borel bijection $\phi$ between $X$ and the Cantor set $K$ and then considering the shift action on $K^{\Gamma}$ with the invariant measure $\Phi \circ \mu$.

For positive numbers $p_{1}, \ldots, p_{d}$ with

$$
\sum_{i=1}^{d} p_{i}=1
$$

let $\kappa\left(p_{1}, \ldots, p_{d}\right)$ denote the probability space on $\{1, \ldots, d\}$ where $P(i)=p_{i}$.

For any $T \subseteq \Gamma$ there is a natural projection $\pi_{T}$ from $\{1, \ldots, d\}^{\Gamma}$ to $\{1, \ldots, d\}^{T}$. The sets of the form $\pi_{T}^{-1}(D)$ with $D \subseteq\{1, \ldots, d\}^{T}$ are called the cylinder sets with respect to $T$.

Lemma 5 Let $f$ be a p.m.p. action of the countable group $\Gamma$ on $(X, \mathcal{B}, \mu)$. Then $f$ weakly contains $\kappa\left(p_{1}, \ldots, p_{d}\right)^{\Gamma}$ if for all finite subsets $F$ of $\Gamma$ and $\delta>0$ there exists a Borel map $\phi: X \rightarrow\{1, \ldots, d\}$ such that for all $\alpha \in\{1, \ldots, d\}^{F}$

$$
\mid \mu\left(\left\{x \in X \mid \phi\left(f_{\gamma} x\right)=\alpha(\gamma) \text { for all } \gamma \in F\right\}\right)-\prod_{\gamma \in F} p_{\alpha(\gamma)} \mid<\delta .
$$

Proof. Assume that the condition holds. Let $g$ denote the shift action of $\Gamma$ on $\kappa\left(p_{1}, \ldots, p_{d}\right)^{\Gamma}$ and let $\lambda$ denote the measure on $\kappa\left(p_{1}, \ldots, p_{d}\right)^{\Gamma}$.

Let $Y_{1}, \ldots, Y_{n} \subseteq\{1, \ldots, d\}^{\Gamma}$ be Borel subsets, let $S \subseteq \Gamma$ be a finite symmetric set containing the identity and let $\varepsilon>0$.

Then for any $r>0$, there exists a finite subset $T$ of $\Gamma$, and subsets $D_{i} \subseteq$ $\{1, \ldots, d\}^{T}$ such that for all $1 \leq i \leq n$ we have

$$
\lambda\left(Y_{i} \triangle \pi_{T}^{-1}\left(D_{i}\right)\right)<r .
$$

Here $\triangle$ denotes symmetric difference. We now apply the condition of the lemma for $F=T S$ and a $\delta>0$ to be chosen later.

For $1 \leq i \leq n$ let

$$
X_{i}=\Phi^{-1}\left(\pi_{T}^{-1}\left(D_{i}\right)\right)
$$


where $\Phi$ is the map defined by 1) in the Introduction. Then for sufficiently small $r$ and $\delta$ it follows that

$$
\left|\mu\left(f_{\gamma} X_{i} \cap X_{j}\right)-\lambda\left(g_{\gamma} Y_{i} \cap Y_{j}\right)\right|<\varepsilon \quad(1 \leq i, j \leq n, \gamma \in S) .
$$

The lemma is proved.

Lemma 6 Let $f$ be a free p.m.p. action of the countable group $\Gamma$ on the compact metric space $(X, d, \mu)$. Then for any finite symmetric subset $F$ of $\Gamma$ and any $\varepsilon>0$ there exists $s>0$ such that

$$
\mu\left(\left\{x \in X \mid d\left(f_{\gamma} x, f_{\gamma^{\prime}} x\right)>s \text { for all } \gamma \neq \gamma^{\prime} \in F\right\}\right)>1-\varepsilon
$$

and

$$
\mu \times \mu\left(\left\{\left(x, x^{\prime}\right) \in X \times X \mid d\left(f_{\gamma} x, f_{\gamma^{\prime}} x^{\prime}\right)>s \text { for all } \gamma, \gamma^{\prime} \in F\right\}\right)>1-\varepsilon
$$

In other terms, for this $s$, most of the $F$-neighbourhoods of pairs of points in $X$ are $s$-apart. We leave the details to the reader.

We are ready to prove Theorem 1 .

Proof of Theorem 1. We will verify that the conditions of Lemma 5 hold. Let $F$ be a finite subset of $\Gamma$ and let $\delta>0$. Let $\varepsilon>0$ be determined later. Use Lemma 6 with this $\varepsilon$ to find $s$ that satisfies the conclusions of the lemma. Let $\left\{B_{j} \mid 1 \leq j \leq J\right\}$ be a Borel partition of $X$ into sets of diameter less than $s$. Let $U_{1}, \ldots, U_{J}$ be independent random variables with distribution $\kappa\left(p_{1}, \ldots, p_{d}\right)$. Define a random map $\phi: X \rightarrow\{1, \ldots, d\}$ by setting

$$
\phi(x)=U_{j} \text { when } x \in B_{j} .
$$

We shall show that with high probability, this map will satisfy the conditions of Lemma 5. In particular, this will imply the existence of such a map.

We shall use the so-called second moment method. For $\alpha \in\{1, \ldots, d\}^{F}$ let

$$
G_{\alpha}=\left\{x \in X \mid \phi\left(f_{\gamma} x\right)=\alpha(\gamma) \text { for all } \gamma \in F\right\} .
$$

Now $\mu\left(G_{\alpha}\right)$ is a random variable with expected value given by

$$
\boldsymbol{E} \mu\left(G_{\alpha}\right)=\boldsymbol{E} \int_{X} 1_{G_{\alpha}}(x) d \mu(x)=\int_{X} \boldsymbol{E} 1_{G_{\alpha}}(x) d \mu(x) .
$$

For $x \in X$ such that the $F$-neighbourhood of $x$ is $s$-apart (that is, the set defined in $\left(^{*}\right)$ of Lemma 6) the expected value $\boldsymbol{E} 1_{G_{\alpha}}(x)=\prod_{\gamma \in F} p_{\alpha(\gamma)}$ because the $\phi$-values at the neighbours are independent. It follows that

$$
\left|\boldsymbol{E} \mu\left(G_{\alpha}\right)-\prod_{\gamma \in F} p_{\alpha(\gamma)}\right|<2 \varepsilon
$$


We shall now estimate the variance of $\mu\left(G_{\alpha}\right)$. We have

$$
\begin{aligned}
\boldsymbol{E} \mu^{2}\left(G_{\alpha}\right) & =\boldsymbol{E} \int_{X} 1_{G_{\alpha}}(x) d \mu(x) \int_{X} 1_{G_{\alpha}}\left(x^{\prime}\right) d \mu\left(x^{\prime}\right)= \\
& =\boldsymbol{E} \int_{X} \int_{X} 1_{G_{\alpha}}(x) 1_{G_{\alpha}}\left(x^{\prime}\right) d(\mu \times \mu)\left(x, x^{\prime}\right)= \\
& =\int_{X} \int_{X} \boldsymbol{E}\left(1_{G_{\alpha}}(x) 1_{G_{\alpha}}\left(x^{\prime}\right)\right) d(\mu \times \mu)\left(x, x^{\prime}\right)
\end{aligned}
$$

For $x, x^{\prime} \in X$ such that the $F$-neighbourhoods of $x$ and $x^{\prime}$ are $s$-apart (that is, when both $x, x^{\prime}$ satisfy $(*)$ and $\left(x, x^{\prime}\right)$ satisfies $(* *)$ of Lemma 6) then

$$
\boldsymbol{E} 1_{G_{\alpha}}(x) 1_{G_{\alpha}}\left(x^{\prime}\right)=\left(\prod_{\gamma \in F} p_{\alpha(\gamma)}\right)^{2}
$$

because the $\phi$-values at the neighbours are independent. The $\mu \times \mu$-measure of these pairs of points is at least $1-3 \varepsilon$. It follows that

$$
\operatorname{Var}\left(\mu\left(G_{\alpha}\right)\right)=\boldsymbol{E} \mu^{2}\left(G_{\alpha}\right)-\left(\boldsymbol{E} \mu\left(G_{\alpha}\right)\right)^{2}<12 \varepsilon .
$$

Now Chebyshev's inequality says that the probability

$$
P\left(\left|\mu\left(G_{\alpha}\right)-\boldsymbol{E} \mu\left(G_{\alpha}\right)\right|>c\right) \leq \frac{\operatorname{Var}\left(\mu\left(G_{\alpha}\right)\right)}{c^{2}}
$$

Setting $c=\varepsilon^{1 / 3}$ we get

$$
P\left(\left|\mu\left(G_{\alpha}\right)-\boldsymbol{E} \mu\left(G_{\alpha}\right)\right|>\varepsilon^{1 / 3}\right) \leq 12 \varepsilon^{1 / 3}
$$

Setting $\varepsilon$ small enough, we get that $\left|\mu\left(G_{\alpha}\right)-\boldsymbol{E} \mu\left(G_{\alpha}\right)\right|$ is arbitrarily small for all $\alpha \in\{1, \ldots, d\}^{F}$ simultaneously. Hence the conditions of Lemma 5 hold and $f$ weakly contains $\kappa\left(p_{1}, \ldots, p_{d}\right)^{\Gamma}$ for any finite distribution $\kappa\left(p_{1}, \ldots, p_{d}\right)$.

Since any probability distribution can be approximated by finite distributions, we get that $f$ weakly contains all Bernoulli actions and the theorem has been established.

\section{Groupoid cost and weak containment}

In this section we will introduce the groupoid cost of a p.m.p. action and show that for finitely generated groups, it is monotonic with respect to weak containment. Groupoid cost has been introduced in [1] as an extension of cost for non-free p.m.p. actions. The monotonicity result was proved by A. Kechris [7. Corollary 10.14] for the original cost and free actions.

Let $f$ be a p.m.p. action of the countable group $\Gamma$ on $(X, \mathcal{B}, \mu)$. Without loss of generality, we can assume that the space $X$ is compact. Endow $\Gamma$ with 
the discrete topology and the counting measure. Consider $X \times \Gamma$, endowed with the product Borel structure and the product measure $\widetilde{\mu}$. We define a partial product on $X \times \Gamma$ as follows. The product of $\left(x_{1}, \gamma_{1}\right)$ and $\left(x_{2}, \gamma_{2}\right)$ is only defined, when $f_{\gamma_{1}} x_{1}=x_{2}$; in this case let $\left(x_{1}, \gamma_{1}\right) \cdot\left(x_{2}, \gamma_{2}\right)=\left(x_{1}, \gamma_{1} \gamma_{2}\right)$. The inverse of $(x, \gamma)$ is defined as $\left(f_{\gamma} x, \gamma^{-1}\right)$ and is denoted by $(x, \gamma)^{-1}$, so we have $(x, \gamma) \cdot(x, \gamma)^{-1}=(x, e)$. Then $X \times \Gamma$ forms a groupoid with respect to this partial product.

Now we define the subset groupoid $\mathcal{G}_{f}$ as follows. Elements of $\mathcal{G}_{f}$ are Borel subsets of $X \times \Gamma$. For $A, B \in \mathcal{G}_{f}$ let

$$
A \cdot B=\{a \cdot b \mid a \in A, b \in B \text { when } a \cdot b \text { is defined }\} \text {. }
$$

Let $E=X \times\{e\}$ where $e$ is the identity element of $\Gamma$. Then for all $A \in \mathcal{G}_{f}$ we have $A E=E A=A$. When $f$ is a continuous action on a compact space, the partial product is continuous as well, which in particular implies that the product of open elements of $\mathcal{G}_{f}$ is open and the product of compact elements is compact.

We say that $A \in \mathcal{G}_{f}$ generates the action $f$, if

$$
\bigcup_{n=1}^{\infty}\left(A \cup A^{-1} \cup E\right)^{n}=X \times \Gamma .
$$

In particular, for any generating set $S$ of $\Gamma, X_{S}=X \times S$ generates $f$. Note that this is a topological condition and is independent of the measure.

The groupoid cost of $f$ is defined as

$$
\operatorname{gcost}(f)=\inf _{A \text { generates } f} \widetilde{\mu}(A)
$$

When $f$ is a free action, $X \times \Gamma$ can be identified with the equivalence relation generated by $f$ on $X$, elements of $\mathcal{G}_{f}$ are graphings in this relation and $A \in \mathcal{G}_{f}$ generates $f$ if and only if the correspoding graphing generates the equivalence relation. Hence, for free actions, $\operatorname{gcost}(f)=\operatorname{cost}(f)$ and so the groupoid cost is indeed an extension of cost to non-free actions. Note that the original notion of cost required that the graphing generates the relation only up to a nullset, but it is easy to see that without loss of generality, one can assume full generation.

Trivially, for every generator $A \in \mathcal{G}_{f}$ and $\varepsilon>0$ there exists an open generator $B \in \mathcal{G}_{f}$ such that $A \subseteq B$ and $\widetilde{\mu}(B) \leq \widetilde{\mu}(A)+\varepsilon$. So, in the definition of gcost, it is enough to consider open generators. The following lemma is essentially contained in [1].

Lemma 7 Assume that $X$ is compact, totally disconnected and $\Gamma$ is finitely generated. Then

$$
\operatorname{gcost}(f)=\inf \widetilde{\mu}(A)
$$

where $A$ varies over compact, clopen generators for $f$. 
Proof. Let $\Gamma$ be generated by the finite set $S$. Let $\mathcal{C}$ be a countable clopen basis for the topology of $X$. List the elements of $\mathcal{C}$ as $C_{1}, C_{2}, \ldots$ For an open set $O \in \mathcal{B}$ and $n>0$ let

$$
O_{n}=\bigcup_{\substack{1 \leq i \leq n \\ C_{i} \subseteq O}} C_{i} .
$$

Let $A \in \mathcal{G}_{f}$ be an open generating element for $\mathcal{G}_{f}$ with $\widetilde{\mu}(A)<\infty$. Then $A=\cup_{\gamma \in \Gamma} A(\gamma) \times\{\gamma\}$ where $A(\gamma) \times\{\gamma\}=A \cap(X \times\{\gamma\})$ and $A(\gamma)$ is open. For $n>0$ let $A_{n}=\cup_{\gamma \in \Gamma} A(\gamma)_{n} \times\{\gamma\}$. Then $A_{n}$ is compact (since $\left.\widetilde{\mu}(A)<\infty\right)$ and clopen. Let $B_{n}=A_{n} \cup A_{n}^{-1} \cup E$. Then

$$
\bigcup_{n=1}^{\infty} B_{n}^{n}=X \times \Gamma
$$

since every possible finite product in $A \cup A^{-1} \cup E$ is realized in $B_{n}$ for large enough $n$. In particular,

$$
\bigcup_{n=1}^{\infty}\left(B_{n}^{n} \cap X_{S}\right)=X_{S}
$$

but since $B_{n}^{n} \cap X_{S}$ is open and $X_{S}$ is compact, there exists an $n>0$ with $X_{S} \subseteq B_{n}^{n}$. Since $X_{S}$ generates $f$, we get that already $A_{n}$ generates $f$.

It follows that any open generator for $f$ with finite measure contains a compact, clopen generator. The lemma is proved.

For the convenience of the reader, we now recall some definitions from the Introduction. For a compact topological space $K$ let $K^{\Gamma}$ denote the shift of $\Gamma$ with base set $K$. This is a continuous action on a compact space. Let $M(\Gamma, K)$ denote the set of $\Gamma$-invariant Borel measures on $K^{\Gamma}$, endowed with the weak* topology and let $E(\Gamma, K) \subseteq M(\Gamma, K)$ denote the set of ergodic measures.

For an ergodic p.m.p. action $f$ on $(X, \mathcal{B}, \mu)$ any Borel map $\phi: X \rightarrow K$ defines a $\Gamma$-equivariant map $\Phi: X \rightarrow K^{\Gamma}$ by setting, for $x \in X$ and $\gamma \in \Gamma$

$$
\Phi(x)(\gamma)=\phi\left(f_{\gamma}(x)\right) .
$$

The measure $\Phi \circ \mu$ is an invariant measure on $K^{\Gamma}$.

Let

$$
E(f, K)=\{\Phi \circ \mu \mid \phi \text { a Borel map from } X \text { to } K\} .
$$

One can also describe $E(f, K)$ as the set of invariant measures on $K^{\Gamma}$ that are factors of $f$.

Lemma 8 Let $f$ and $g$ be p.m.p. actions of the countable group $\Gamma$ on $(X, \mathcal{B}, \mu)$ and $(Y, \mathcal{C}, \nu)$, respectively. Then $f$ weakly contains $g$ if and only if

$$
\overline{E(f, K)} \supseteq E(g, K)
$$

for any compact topological space $K$. The same result holds if we only consider the class of finite spaces. 
Proof. The $\left({ }^{*}\right)$ condition for all finite spaces $K$ is easily seen to be equivalent with the original definition of weak containment.

If $L$ is a finite subset of $K$, then $L^{\Gamma}$ is contained in $K^{\Gamma}$. The invariant measures concentrated on $L^{\Gamma}$ as $L$ varies over the finite subsets of $K$, are dense in $M(\Gamma, K)$.

Moreover, it follows that

$$
\overline{\bigcup_{L \subseteq K \text { finite }} E(g, L)} \supseteq E(g, K) .
$$

This is because

$$
E(g, K)=\{\Phi \circ \nu \mid \phi \text { a Borel map from } Y \text { to } K\} .
$$

and the Borel map $\phi$ can be well approximated by maps $\phi_{n}$ with finite range $L_{n}$ in such a way that $\Phi_{n} \circ \nu$ converges to $\Phi \circ \nu$. This yields

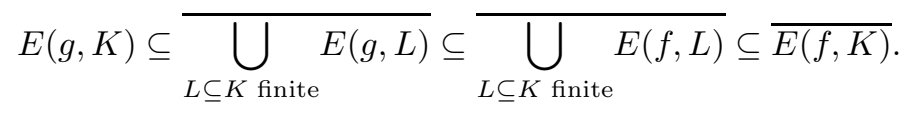

The lemma is proved.

Theorem 9 Let $f$ and $g$ be p.m.p. actions of the finitely generated group $\Gamma$ on $(X, \mathcal{B}, \mu)$ and $(Y, \mathcal{C}, \nu)$, respectively, such that $f$ weakly contains $g$. Then $\operatorname{gcost}(f) \leq \operatorname{gcost}(g)$.

Proof. Let $K$ be the standard Cantor set. Let $\phi$ be a Borel isomorphism from $Y$ to $K$. Then the shift action on $K^{\Gamma}$ with the invariant measure $\nu^{\prime}=\Phi \circ \nu$ is isomorphic to $g$. Let $h$ be the shift action of $\Gamma$ on $K^{\Gamma}$ with the invariant measure $\nu^{\prime}$. Clearly, gcost $(h)=\operatorname{gcost}(g)$. Using Lemma 7 , for any $\varepsilon>0$ there exists a clopen, compact $A \in \mathcal{G}_{h}$ that generates $h$ and $\widetilde{\nu}^{\prime}(A)<\operatorname{gcost}(h)+\varepsilon$. Since weak convergence of probability measures $\rho_{n}$ on $K^{\Gamma}$ implies convergence of $\widetilde{\rho_{n}}$ on clopen compact subsets of $K^{\Gamma} \times \Gamma$, using Lemma 8 there exists a map $\psi: X \rightarrow K$ such that

$$
\widetilde{\Psi \circ \mu}(A) \leq \widetilde{\nu}^{\prime}(A)+\varepsilon .
$$

The condition of generating is independent of the invariant measure, so $A$ generates the shift action on $K^{\Gamma}$ with the invariant measure $\Psi \circ \mu$ as well. This action is a factor of $f$, and hence its gcost is an upper bound for the gcost of $f$. This implies

$$
\operatorname{gcost}(f) \leq \widetilde{\Psi \circ \mu}(A) \leq \widetilde{\nu}^{\prime}(A)+\varepsilon<\operatorname{gcost}(g)+2 \varepsilon .
$$

The theorem holds.

Proof of Corollary 2, Let $\Gamma$ be a finitely generated group and let $f$ be a free p.m.p. action of $\Gamma$. Then, using Theorem 1, $f$ weakly contains all Bernoulli actions, so by Theorem 9 the gcost of $f$ is at most the gcost of any Bernoulli 
action of $\Gamma$, in particular, the cost of $f$ is at most the cost of any free Bernoulli action of $\Gamma$.

Note that any nontrivial Bernoulli action of an infinite group is free. Also, it is easy to see that when $\Gamma$ is torsion free, then every nontrivial factor of i.i.d. is free. However, in the presence of torsion, this last statement fails to hold.

\section{Ergodic actions and convexity}

Let $f$ be a p.m.p. action of the countable group $\Gamma$ on $(X, \mathcal{B}, \mu)$. A sequence of subsets $A_{n} \in \mathcal{B}$ is almost invariant, if

$$
\lim _{n \rightarrow \infty} \mu\left(f_{\gamma} A_{n} \triangle A_{n}\right)=0 \text { for all } \gamma \in \Gamma .
$$

The sequence is trivial, if $\lim _{n \rightarrow \infty} \mu\left(A_{n}\right)\left(1-\mu\left(A_{n}\right)\right)=0$. We say that the action $f$ is strongly ergodic, if every almost invariant sequence is trivial.

The following lemma is due to K. Schmidt 9 .

Lemma 10 (Schmidt) Let $f$ be a p.m.p. action of the countable group $\Gamma$ on $(X, \mathcal{B}, \mu)$, such that $f$ is ergodic, but not strongly ergodic. Then for all $0<\lambda<1$ there exists an almost invariant sequence $A_{n}$ such that $\mu\left(A_{n}\right)=\lambda(n \geq 0)$.

In particular, the action is strongly ergodic if and only if it is ergodic and does not weakly contain the identity action of $\Gamma$ on two points with equal mass.

The following lemma forms a part of the proof of Schmidt's lemma. For completeness, we provide a short proof.

Lemma 11 Let $f$ be an ergodic p.m.p. action of the countable group $\Gamma$ on the compact space $(X, \mathcal{B}, \mu)$, let $0<\lambda<1$ and let $A_{n}$ be an almost invariant sequence such that

$$
\lim _{n \rightarrow \infty} \mu\left(A_{n}\right)=\lambda .
$$

Let $\mu_{n}$ be the measure on $X$ defined by

$$
\mu_{n}(Y)=\mu\left(A_{n} \cap Y\right) \quad(Y \in \mathcal{B}) .
$$

Then $\mu_{n}$ weak $^{*}$ converges to $\lambda \mu$.

Proof. Let $n_{k}$ be a sequence such that $\mu_{n_{k}}$ is weakly convergent and let $\nu$ be the limit measure. Then $\nu$ is invariant under $\Gamma$ and is bounded by $\mu / \lambda$, so by ergodicity, $\nu$ is a scalar multiple of $\mu$. But $\nu(X)=\lim \mu\left(A_{n}\right)=\lambda$ which proves the lemma.

Proof of Theorem 3. Let $f$ be an ergodic p.m.p. action of the countable group $\Gamma$ on $(X, \mathcal{B}, \mu)$.

Assume that $f$ is weakly equivalent to $f \times I$. Since $f \times I$ factors on the identity action $b$ of $\Gamma$ on two points with equal mass, this means that $f$ weakly 
contains $b$. Hence $f$ can not be strongly ergodic.

Now assume $f$ is not strongly ergodic. We claim that $f$ weakly contains $f \times b$. We can assume that the space $(X, \mathcal{B}, \mu)$ is totally disconnected and compact and $\Gamma$ acts by continuous maps. Let $\mathcal{C}$ denote the set of clopen sets in $\mathcal{B}$. Then $\mathcal{C}$ is a finitely additive algebra and for any $B \in \mathcal{B}$ and $\varepsilon>0$ there exists $C \in \mathcal{C}$ with $\mu(B \triangle C)<\varepsilon$.

Hence, it is enough to check the weak containment condition for clopen subsets. In addition, by Schmidt's lemma, there exists an almost invariant sequence $A_{n} \in \mathcal{C}$ with

$$
\lim _{n \rightarrow \infty} \mu\left(A_{n}\right)=1 / 2 .
$$

Lemma 11 implies that for every clopen set $C \in \mathcal{C}$, we have

$$
\lim _{n \rightarrow \infty} \mu\left(C \cap A_{n}\right)=\mu(C) / 2 .
$$

Indeed, weak* convergence of measures implies convergence on clopen sets.

Let $Y_{1}^{\prime}, \ldots, Y_{k}^{\prime}, Y_{1}^{\prime \prime}, \ldots, Y_{k}^{\prime \prime} \in \mathcal{C}$, let $S \subseteq \Gamma$ be a finite subset and let $\varepsilon>0$. Then by the almost invariance of $A_{n}$, for all $\gamma \in S$ we have

$$
\lim _{n \rightarrow \infty} \mu\left(f_{\gamma} A_{n} \triangle A_{n}\right)=0
$$

Also for all $1 \leq i, j \leq k$ and $\gamma \in S$ we have

$$
\lim _{n \rightarrow \infty} \mu\left(A_{n} \cap f_{\gamma} Y_{i}^{\prime} \cap Y_{j}^{\prime}\right)-\mu\left(f_{\gamma} Y_{i}^{\prime} \cap Y_{j}^{\prime}\right) / 2=0
$$

and

$$
\lim _{n \rightarrow \infty} \mu\left(A_{n} \cap f_{\gamma} Y_{i}^{\prime \prime} \cap Y_{j}^{\prime \prime}\right)-\mu\left(f_{\gamma} Y_{i}^{\prime \prime} \cap Y_{j}^{\prime \prime}\right) / 2=0
$$

Let $n$ be large enough such that all the above quantities (listed in the above three displays) are less than $\varepsilon$. Now for $1 \leq i \leq k$ let

$$
X_{i}=\left(A_{n} \cap Y_{i}^{\prime}\right) \cup\left(A_{n}^{c} \cap Y_{i}^{\prime \prime}\right)
$$

where $A_{n}^{c}$ denotes the complement of $A_{n}$.

Now let $Y_{1}, \ldots, Y_{k}$ be Borel subsets of $X \times\{0,1\}$. Let

$$
Y_{i}^{\prime} \times\{0\}=Y_{i} \cap(X \times\{0\})
$$

and let

$$
Y_{i}^{\prime \prime} \times\{1\}=Y_{i} \cap(X \times\{1\}) .
$$

Applying the above argument for $Y_{i}^{\prime}$ and $Y_{i}^{\prime \prime}$ we obtain the desired measurable subsets $X_{1}, \ldots, X_{n}$. The claim holds and $f$ weakly contains $f \times b$.

Iterating the argument, we get that $f$ weakly contains $f \times b_{2^{n}}$ where $b_{2^{n}}$ is the trivial action of $\Gamma$ on $\left\{1, \ldots, 2^{n}\right\}$ with uniform measure. An easy approximation argument yields that $f$ weakly contains $f \times I$. Since $f$ is a factor of $f \times I$, we get that $f$ is weakly equivalent to $f \times I$. 
The theorem is proved.

Proof of Theorem 4. Let $f$ be an ergodic p.m.p. action of the countable group $\Gamma$.

Assume that $f$ is strongly ergodic. Assume by contradiction that there exists a sequence of Borel maps $\phi_{n}: X \rightarrow K$ such that $\Phi_{n} \circ \mu$ weak* converges to a nonergodic measure $\lambda$ on $K^{\Gamma}$. Let $A$ be a $\Gamma$-invariant Borel set with $0<\lambda(A)<1$. Let us approximate $A$ with a clopen sequence $A_{n}$ with respect to $\lambda$. It is easy to see that $A_{n}$ is almost invariant with respect to $\lambda$. Then there exists $k(n)$ such that $\Phi_{k(n)}^{-1}\left(A_{n}\right)$ gives us a nontrivial almost invariant sequence for $f$, a contradiction. Hence $\overline{E(f, K)} \subseteq E(\Gamma, K)$.

Assume that $f$ is not strongly ergodic. Let $\mu^{\prime}$ be the product measure $\mu \times\{1 / 2,1 / 2\}$. Let $\phi_{0}, \phi_{1}: X \rightarrow K$ be Borel maps and let $\phi: X \times\{0,1\} \rightarrow K$ be defined by

$$
\phi((x, i))=\phi_{i}(x) \text { for } i=0,1 .
$$

Then by Theorem 3, $f$ weakly contains $f \times b$, so by Lemma 4, $\Phi \circ \mu^{\prime}$ is in $\overline{E(f, K)}$. But

$$
\Phi \circ \mu^{\prime}=\frac{1}{2} \Phi_{0} \circ \mu+\frac{1}{2} \Phi_{1} \circ \mu .
$$

This gives that for $\lambda, \lambda^{\prime} \in E(f, K), \frac{1}{2} \lambda+\frac{1}{2} \lambda^{\prime} \in \overline{E(f, K)}$. Hence $\overline{E(f, K)}$ is convex.

The theorem holds.

\section{References}

[1] M. AbÉRT And N. Nikolov, Rank gradient, cost of groups and the rank versus Heegard genus problem, to appear in J. of the EMS

[2] A. Connes and B. Weiss, Property T and asymptotically invariant sequences, Israel Journal of Math. 371980 209-210.

[3] D. Gaboriau, Coût des relations d'équivalence et des groupes. (French) [Cost of equivalence relations and of groups] Invent. Math. 139 (2000), no. $1,41-98$.

[4] E. Glasner, J.-P. Thouvenot and B. Weiss, Every countable group has the weak Rohlin property, Bull. London Math. Soc. 38, 2006, 932-936.

[5] E. Glasner And B. Weiss, Kazhdan's property T and the geometry of the collection of invariant measures, Geom. Funct. Anal. 7 (1997), no. 5, 917-935.

[6] V. Jones And K. Schmidt, Asymptotically invariant sequences and approximate finiteness, Am. J. of Math. 109 (1987) 91-114.

[7] A. Kechris, Global aspects of ergodic group actions, to appear in the series "Mathematical Surveys and Monographs" of the AMS 
[8] G. Levite, On the cost of generating an equivalence relation, Ergodic Theory Dynam. Systems 15 (1995), no. 6, 1173-1181.

[9] K. Schmidt, Amenability, Kazhdan's property $T$, strong ergodicity and invariant means for ergodic group-actions, Ergodic Theory Dynamical Systems 1 (1981), no. 2, 223-236.

[10] K. Schmidt, Asymptotically invariant sequences and an action of $S L(2, Z)$ on the 2-sphere, Israel J. of Math. 37 (1980), 193-208. 